\title{
Effect of non-uniform temperature gradient and magnetic field on onset of Marangoni convection heated from below by a constant heat flux
}

\begin{abstract}
This paper investigates the effect of non-uniform temperature gradient and magnetic field on Marangoni convection in a horizontal fluid layer heated from below and cooled from above with a constant heat flux. A linear stability analysis is performed. The influence of various parameters on the convection onset is analyzed. Six non-uniform basic temperature profiles are considered, and some general conclusions about their destabilizing effects are presented.
\end{abstract}

Keyword: Marangoni convection; Non-uniform temperature; Magnetic field; O352; 76E06; 76E25 